\title{
The Novikov conjecture and geometry of Banach spaces
}

\author{
GENNADI KASPAROV \\ GUOLIANG YU
}

In this paper, we prove the strong Novikov conjecture for groups coarsely embeddable into Banach spaces satisfying a geometric condition called Property $(\mathrm{H})$.

19K35, 19K56, 46L80; 57R55

\section{Introduction}

An important problem in higher dimensional topology is the Novikov conjecture on the homotopy invariance of higher signature. The Novikov conjecture is a consequence of the Strong Novikov Conjecture in $K$-theory of group $C^{*}$-algebras. The main purpose of this paper is to prove the Strong Novikov conjecture (with coefficients) for any group coarsely embeddable into a Banach space satisfying a geometric condition called Property (H).

Definition 1.1 A real Banach space $X$ is said to have Property $(H)$ if there exists an increasing sequence of finite dimensional subspaces $\left\{V_{n}\right\}$ of $X$ and an increasing sequence of finite dimensional subspaces $\left\{W_{n}\right\}$ of a real Hilbert space such that

(1) $V=\bigcup_{n} V_{n}$ is dense in $X$;

(2) if $W$ denotes $\bigcup_{n} W_{n}$, and $S(V), S(W)$ denote respectively the unit spheres of $V, W$, then there exists a uniformly continuous map $\psi: S(V) \rightarrow S(W)$ such that the restriction of $\psi$ to $S\left(V_{n}\right)$ is a homeomorphism (or more generally a degree one map) onto $S\left(W_{n}\right)$ for each $n$.

As an example, let $X$ be the Banach space $l^{p}(\mathbb{N})$ for some $p \geq 1$. Let $V_{n}$ and $W_{n}$ be respectively the subspaces of $l^{p}(\mathbb{N})$ and $l^{2}(\mathbb{N})$ consisting of all sequences whose coordinates are zero after the $n$th terms. We define a map $\psi$ from $S(V)$ to $S(W)$ by

$$
\psi\left(c_{1}, \ldots, c_{k}, \ldots\right)=\left(c_{1}\left|c_{1}\right|^{p / 2-1}, \ldots, c_{k}\left|c_{k}\right|^{p / 2-1}, \ldots\right) .
$$

This map $\psi$ is called the Mazur map (see Benyamini and Lindenstrauss [3]). It is not difficult to verify that $\psi$ satisfies the conditions in the definition of Property $(\mathrm{H})$. For 
each $p \geq 1$, we can similarly prove that the Banach space of all Schatten $p$-class operators on a Hilbert space has Property $(\mathrm{H})$. We can also check that uniformly convex Banach spaces with certain unconditional bases have Property $(\mathrm{H})$ (with the help of results of Benyamini and Lindenstrauss on the uniform homeomorphism classification of Banach space spheres in [3, Chapter 9]).

We also recall that a metric space $\Gamma$ is said to be coarsely embeddable into a Banach space $X$ (see Gromov [5]) if there exists a map $h: \Gamma \rightarrow X$ for which there exist non-decreasing functions $\rho_{1}$ and $\rho_{2}$ from $\mathbb{R}_{+}=[0, \infty)$ to $\mathbb{R}$ such that

(1) $\rho_{1}(d(x, y)) \leq\|h(x)-h(y)\| \leq \rho_{2}(d(x, y))$ for all $x, y \in \Gamma$;

(2) $\lim _{r \rightarrow+\infty} \rho_{i}(r)=+\infty$ for $i=1,2$.

In the case of a countable group $\Gamma$, we endow $\Gamma$ with a proper (left invariant) length metric. If $\Gamma$ is finitely generated, the word length metric is an example of a proper length metric. The issue of coarse embeddability of a countable group into a Banach space $X$ is independent of the choice of the proper length metric.

The following theorem is the main result of this paper.

Theorem 1.2 Let $\Gamma$ be a countable discrete group and $A$ any $\Gamma-C^{*}$-algebra. If $\Gamma$ admits a coarse embedding into a Banach space with Property $(H)$, then the Strong Novikov conjecture with coefficients in $A$ holds for $\Gamma$, that is, if $\mathcal{E} \Gamma$ is the universal space for proper $\Gamma$-actions and $A \rtimes_{r} \Gamma$ is the reduced crossed product $C^{*}$-algebra, then the Baum-Connes assembly map

$$
\mu: K K_{*}^{\Gamma}(\mathcal{E} \Gamma, A) \rightarrow K_{*}\left(A \rtimes_{r} \Gamma\right)
$$

is injective.

The special case when the Banach space is the Hilbert space is proved by $\mathrm{Yu}$ [15] and Skandalis-Tu-Yu [13].

If we replace the degree one condition by a nonzero degree condition in the definition of Property (H), we say that $X$ has rational Property (H).

Theorem 1.3 Let $\Gamma$ be a countable discrete group and $A$ any $\Gamma-C^{*}$-algebra. If $\Gamma$ admits a coarse embedding into a Banach space with rational Property $(H)$, then the rational Strong Novikov conjecture with coefficients in $A$ holds for $\Gamma$, that is, if $\mathcal{E} \Gamma$ is the universal space for proper $\Gamma$-actions and $A \rtimes_{r} \Gamma$ is the reduced crossed product $C^{*}$-algebra, then the Baum-Connes assembly map

$$
\mu: K K_{*}^{\Gamma}(\mathcal{E} \Gamma, A) \otimes \mathbb{Q} \rightarrow K_{*}\left(A \rtimes_{r} \Gamma\right) \otimes \mathbb{Q}
$$

is injective. 
We remark that the rational Strong Novikov conjecture implies the Novikov conjecture on homotopy invariance of higher signatures and the Gromov-Lawson-Rosenberg conjecture regarding nonexistence of positive scalar curvature metrics on closed aspherical manifolds.

It is conjectured that any countable subgroup of the diffeomorphism group of a compact smooth manifold is coarsely embeddable into the Banach space of all Schatten $p$-class operators for some $p \geq 1$. If $p>2$, then $l^{p}(\mathbb{N})$ is not coarsely embeddable into a Hilbert space (see Johnson and Randrianarivony [9]). More generally, $l^{p}(\mathbb{N})$ does not coarsely embed into $l^{q}(\mathbb{N})$ if $p>q \geq 2$ (see Mendel and Naor [11]). Let $C_{0}$ be the Banach space consisting of all sequences of real numbers that are convergent to 0 . It is an open question if $C_{0}$ has (rational) Property $(\mathrm{H})$. By the above theorems, a positive answer to this question would imply the Novikov conjecture since every countable group admits a coarse embedding into $C_{0}$ (see Brown and Guentner [4]).

This paper is organized as follows. In Section 2, we construct a $C^{*}$-algebra associated to a Banach space with (rational) Property $(\mathrm{H})$ and study its $K$-groups. In Section 3, we reformulate the Baum-Connes map and discuss its connection with the localization algebra. In Section 4, we introduce the Bott map for $K$-groups. In Section 5, we give a proof of the main theorem.

In this paper, $K$-groups of a graded $C^{*}$-algebra are defined to be the $K$-groups of the underlying ungraded $C^{*}$-algebra obtained by forgetting the grading structure. The same comment applies to $K K$-groups.

Acknowledgements The authors wish to thank Misha Gromov for inspiring discussions and the referee for very helpful comments. Both authors are partially supported by NSF.

\section{A $C^{*}$-algebra associated to a Banach space with Property (H)}

In this section, we construct a $C^{*}$-algebra associated to a Banach space with (rational) Property $(\mathrm{H})$ and study its $K$-groups.

Let $\psi$ be as in the definition of (rational) Property $(\mathrm{H})$. We extend $\psi$ to a map $\phi: V \rightarrow W$ by

$$
\phi(v)=\|v\| \psi\left(\frac{v}{\|v\|}\right)
$$

for any $v \in V$, where $\phi(0)$ should be interpreted as 0 . 
Let Clifford $\left(W_{n}\right)$ be the complex Clifford algebras of $W_{n}$, satisfying the relation $w^{2}=\|w\|^{2}$ for all $w \in W_{n}$. We define the complex Clifford algebra Clifford $(W)$ to be the $C^{*}$-algebra inductive limit of $\operatorname{Clifford}\left(W_{n}\right)$. Let $C_{0}(V, \operatorname{Clifford}(W))$ be the graded $C^{*}$-algebra of all bounded and uniformly continuous functions on $V$ with values in the Clifford algebra $\operatorname{Clifford}(W)$ which vanish at infinity, where the grading is given by the natural grading structure on the Clifford algebra. Let $\mathcal{S}=C_{0}(\mathbb{R})$ be the graded $C^{*}$-algebra of all complex-valued continuous functions on $\mathbb{R}$ vanishing at infinity (graded by even and odd functions). We define $\mathcal{S} \hat{\otimes} C_{0}(V, \operatorname{Clifford}(W))$ to be the graded $C^{*}$-algebra tensor product of $\mathcal{S}$ and $C_{0}(V, C l i f f o r d(W))$.

For any $f \in C_{0}(\mathbb{R})$, we can define an element

$$
f((s, \phi(v))) \in \mathcal{S} \hat{\otimes} C_{0}(V, \operatorname{Clifford}(W))
$$

by

$$
f((s, \phi(v)))=f(s \hat{\otimes} 1+1 \hat{\otimes} \phi(v)),
$$

where $s$ and $\phi(v)$ should be respectively viewed as unbounded degree one multipliers of $\mathcal{S}$ and $C_{0}(V, \operatorname{Clifford}(W)),(s, \phi(v))$ is defined to be $s \hat{\otimes} 1+1 \hat{\otimes} \phi(v)$ as an unbounded degree one multiplier of $\mathcal{S} \hat{\otimes} C_{0}(V, \operatorname{Clifford}(W))$, and $f(s \hat{\otimes} 1+1 \hat{\otimes} \phi(v))$ is defined using functional calculus.

More concretely, $f((s, \phi(v)))$ can be defined as follows:

(1) If $f(t)=g\left(t^{2}\right)$ for some $g \in C_{0}(\mathbb{R})$, then we define a scalar valued function $f((s, \phi(v)))$ of the variable $(s, v) \in \mathbb{R} \times V$ by

$$
(s, v) \rightarrow g\left(s^{2}+\|\phi(v)\|^{2}\right)=g\left(s^{2}+\|v\|^{2}\right)
$$

for every $(s, v) \in \mathbb{R} \times V$;

(2) if $f(t)=\operatorname{tg}\left(t^{2}\right) \in C_{0}(\mathbb{R})$ for some $g \in C_{0}(\mathbb{R})$, then we define an element $f((s, \phi(v))) \in \mathcal{S} \hat{\otimes} C_{0}(V, \operatorname{Clifford}(W))$ by

$$
\begin{aligned}
f((s, \phi(v))) & =g\left(s^{2}+\|\phi(v)\|^{2}\right)(s \hat{\otimes} 1+1 \hat{\otimes} \phi(v)) \\
& =g\left(s^{2}+\|v\|^{2}\right)(s \hat{\otimes} 1+1 \hat{\otimes} \phi(v))
\end{aligned}
$$

for every $s \in \mathbb{R}, v \in V$, here $s \hat{\otimes} 1+1 \hat{\otimes} \phi(v)$ should viewed as an unbounded degree one multiplier of $\mathcal{S} \hat{\otimes} C_{0}(V, \operatorname{Clifford}(W))$;

(3) the general definition of $f((s, \phi(v)))$ follows using approximation of $f$ by linear combinations of special functions of the above two types in $C_{0}(\mathbb{R})$.

Now we are ready to define a $C^{*}$-algebra associated to a Banach space with (rational) Property (H). 
Definition 2.1 Let $X$ be a Banach space with (rational) Property $(\mathrm{H})$. Let $\phi$ be as above. We define $\mathcal{A}(X)$ to be the graded $C^{*}$-subalgebra of $\mathcal{S} \hat{\otimes} C_{0}(V, \operatorname{Clifford}(W))$ generated by all $f\left(\left(s, \phi\left(v-v_{0}\right)\right)\right)$ for $s \in \mathbb{R}$, all $v_{0} \in V$ and $f \in C_{0}(\mathbb{R})$.

It is not difficult to compute $K_{*}(\mathcal{A}(X))$ when $X$ is an $l^{p}$-space for some $1 \leq p<\infty$ (see Higson-Kasparov-Trout [8] for $p=2$ ). In general, it is an open question how to compute $K_{*}(\mathcal{A}(X))$. The following result provides a partial solution.

Proposition 2.2 Let $X$ be a Banach space with Property $(H)$ and let $\mathcal{A}(X)$ be the $C^{*}$-algebra associated to $X$. If $B$ is a (graded) $C^{*}$-algebra, then the homomorphism from $\mathcal{S}$ to $\mathcal{A}(X): f(s) \rightarrow f((s, \phi(v))$, induces an injection

$$
K_{*}(\mathcal{S} \hat{\otimes} B) \rightarrow K_{*}(\mathcal{A}(X) \hat{\otimes} B) .
$$

Proof Let $\mathcal{A}\left(X, V_{n}\right)$ be the $C^{*}$-subalgebra of $\mathcal{A}(X)$ generated by all elements $f\left(\left(s, \phi\left(v-v_{0}\right)\right)\right)$ for $f \in C_{0}(\mathbb{R})$ and $s \in \mathbb{R}, v_{0} \in V_{n}$. Note that $\mathcal{A}(X) \hat{\otimes} B$ is the inductive limit of $\mathcal{A}\left(X, V_{n}\right) \hat{\otimes} B$. It suffices to prove that the homomorphism

$$
\beta_{n}: K_{*}(\mathcal{S} \hat{\otimes} B) \rightarrow K_{*}\left(\mathcal{A}\left(X, V_{n}\right) \hat{\otimes} B\right)
$$

is injective for each $n$, where $\beta_{n}$ is induced by the homomorphism from $\mathcal{S}$ to $\mathcal{A}\left(X, V_{n}\right)$ : $f(t) \rightarrow f((s, \phi(v))$.

Let $\mathcal{C}\left(V_{n}\right)$ be the graded $C^{*}$-algebra of all continuous functions on $V_{n}$ with values in the Clifford algebra Clifford $\left(W_{n}\right)$ which vanish at infinity. Define $\mathcal{A}\left(V_{n}\right)$ to be the graded $C^{*}$-algebra tensor product $\mathcal{S} \hat{\otimes} \mathcal{C}\left(V_{n}\right)$. Let $r_{n}$ be the restriction homomorphism from $\mathcal{A}\left(X, V_{n}\right) \hat{\otimes} B$ to $\mathcal{A}\left(V_{n}\right) \hat{\otimes} B$. By the definition of Property $(\mathrm{H})$, such restriction homomorphism is well defined. By Bott periodicity (see Higson-Kasparov-Trout [8]) and the degree one condition in the definition of Property $(\mathrm{H})$, we observe that the composition $\left(r_{n}\right)_{*} \circ \beta_{n}$ is an isomorphism. Proposition 2.2 follows from this observation.

The following result follows from Proposition 2.2 and the Green-Julg theorem.

Corollary 2.3 Let $X$ be a Banach space with Property $(H)$ and let $\mathcal{A}(X)$ be the $C^{*}$-algebra associated to $X$. If $H$ is a compact topological group and $B$ is a (graded) $H-C^{*}$-algebra, then we have a natural injective homomorphism

$$
K_{*}^{H}(\mathcal{S} \hat{\otimes} B) \rightarrow K_{*}^{H}(\mathcal{A}(X) \hat{\otimes} B),
$$

where $H$ acts on $\mathcal{A}(X)$ trivially.

We can prove the following results using essentially the same argument. 
Proposition 2.4 Let $X$ be a Banach space with rational Property $(H)$ and let $\mathcal{A}(X)$ be the $C^{*}$-algebra associated to $X$. If $B$ is a (graded) $C^{*}$-algebra, then we have a natural injective homomorphism

$$
K_{*}(\mathcal{S} \hat{\otimes} B) \otimes \mathbb{Q} \rightarrow K_{*}(\mathcal{A}(X) \hat{\otimes} B) \otimes \mathbb{Q} .
$$

Corollary 2.5 Let $X$ be a Banach space with rational Property $(H)$ and let $\mathcal{A}(X)$ be the $C^{*}$-algebra associated to $X$. If $H$ is a compact topological group and $B$ is a (graded) $H-C^{*}$-algebra, then we have a natural injection

$$
K_{*}^{H}(\mathcal{S} \hat{\otimes} B) \otimes \mathbb{Q} \rightarrow K_{*}^{H}(\mathcal{A}(X) \hat{\otimes} B) \otimes \mathbb{Q} .
$$

Let $B$ be a graded $C^{*}$-algebra, and let $\mathcal{K}$ be the graded $C^{*}$-algebra of all compact operators on a graded separable and infinite dimensional Hilbert space. Let $C_{c}(\mathbb{R}) \hat{\otimes}_{\text {alg }}$ $(B \hat{\otimes} \mathcal{K})$ be the algebraic graded tensor product of $C_{c}(\mathbb{R})$ with $B \hat{\otimes} \mathcal{K}$, where $C_{c}(\mathbb{R})$ is considered as an algebra graded by even and odd functions.

We define $\mathcal{S}_{b}^{\infty}(B)$ to be the graded $C^{*}$-algebra of all bounded sequences of uniformly equicontinuous functions $\left\{f_{k}\right\}$ in $\mathcal{S} \widehat{\otimes} B \widehat{\otimes} \mathcal{K}$ such that for each $\epsilon>0$, there exists $R>0$ for which there exists a bounded sequence of uniformly equicontinuous functions $\left\{g_{k}\right\}$ in $C_{c}(\mathbb{R}) \widehat{\otimes}_{\text {alg }}(B \hat{\otimes} \mathcal{K})$ satisfying diameter(support $\left.\left(g_{k}\right)\right)<R$ and $\left\|f_{k}-g_{k}\right\|<\epsilon$ for all $k$, where the support of $g_{k}$ is a subset of $\mathbb{R}$. Let $\mathcal{S}_{0}^{\infty}(B)$ be the graded $C^{*}$-subalgebra of $\mathcal{S}_{b}^{\infty}(B)$ consisting of all sequence $\left(f_{k}\right)$ in $\mathcal{S}_{b}^{\infty}(B)$ which are convergent to 0 in the sup norm. Define $\mathcal{S}^{\infty}(B)$ to be the quotient graded $C^{*}$-algebra $\mathcal{S}_{b}^{\infty}(B) / \mathcal{S}_{0}^{\infty}(B)$.

The proof of the following result is straightforward and is therefore omitted.

Proposition 2.6 If $H$ is a finite group and $B$ is a graded $H-C^{*}$-algebra, then $K_{*}^{H}\left(\mathcal{S}^{\infty}(B)\right)$ is naturally isomorphic to $\left(\prod K_{*+1}^{H}(B)\right) /\left(\bigoplus K_{*+1}^{H}(B)\right)$.

We remark that by the Green-Julg theorem the equivariant case of the above proposition follows from the non-equivariant case.

Let $\mathcal{C}\left(V_{k}\right)$ be the graded $C^{*}$-algebra of all continuous functions on $V_{k}$ with values in the Clifford algebra Clifford $\left(W_{k}\right)$ which vanish at infinity. Define $\mathcal{A}\left(V_{n}\right)$ to be the graded $C^{*}$-algebra tensor product $\mathcal{S} \hat{\otimes} \mathcal{C}\left(V_{n}\right)$. Endow $\mathbb{R} \times V_{k}$ with the product metric of the standard metric on $\mathbb{R}$ and the Banach norm metric on $V_{k}$.

We define $\mathcal{A}_{b}^{\infty}(X, B)$ to be the graded $C^{*}$-algebra of all bounded sequences $\left(a_{k}\right)$ such that 
(1) $a_{k} \in \mathcal{A}\left(V_{k}\right) \hat{\otimes} B \hat{\otimes} \mathcal{K}$ and $\left(a_{k}\right)$ is uniformly equicontinuous in $\left\{\left(s, v_{k}\right)\right\}$ in the sense that for any $\epsilon>0$ there exists a $\delta>0$ (independent of $k$ ) such that

$$
\left\|a_{k}\left(s, v_{k}\right)-a_{k}\left(s^{\prime}, v_{k}^{\prime}\right)\right\|<\epsilon
$$

if $d\left(\left(s, v_{k}\right),\left(s^{\prime}, v_{k}^{\prime}\right)\right)<\delta$;

(2) for each $\epsilon>0$, there exists $R>0$ for which there exists a sequence $\left\{g_{k}\right\}$ in $\mathcal{A}\left(V_{k}\right) \hat{\otimes} B \widehat{\otimes} \mathcal{K}$ satisfying

$$
\begin{aligned}
\text { diameter(support } \left.\left(g_{k}\right)\right) & <R, \\
\left\|a_{k}\left(s, v_{k}\right)-g_{k}\left(s, v_{k}\right)\right\| & <\epsilon
\end{aligned}
$$

for all $k$ and $\left(s, v_{k}\right) \in \mathbb{R} \times V_{k}$, where the support of $g_{k}$ is a subset of $\mathbb{R} \times V_{k}$.

Let $\mathcal{A}_{0}^{\infty}(X, B)$ be the graded $C^{\infty}$-subalgebra of $\mathcal{A}_{b}^{\infty}(X, B)$ consisting of all sequences which are convergent to 0 in norm. Define $\mathcal{A}^{\infty}(X, B)$ to be the quotient graded $C^{*}$-algebra $\mathcal{A}_{b}^{\infty}(X, B) / \mathcal{A}_{0}^{\infty}(X, B)$.

Let $\beta_{k}$ be the homomorphism from $\mathcal{S}$ to $\mathcal{A}\left(V_{k}\right)$ defined by

$$
\left.\left(\beta_{k}(f)\right)\left(s, v_{k}\right)\right)=f\left(\left(s, \phi\left(v_{k}\right)\right)\right)
$$

for each $f \in \mathcal{S}, s \in \mathbb{R}$ and $v_{k} \in V_{k}$.

$\beta_{k}$ induces a homomorphism (still denoted by $\beta_{k}$ ) from $\mathcal{S} \hat{\otimes} B \hat{\otimes} \mathcal{K}$ to $\mathcal{A}\left(V_{k}\right) \hat{\otimes} B \hat{\otimes} \mathcal{K}$. We define a homomorphism

$$
\beta: \mathcal{S}^{\infty}(B) \rightarrow \mathcal{A}^{\infty}(X, B)
$$

by

$$
\beta\left(\left[\left(f_{1}, \ldots, f_{k}, \ldots\right)\right]\right)=\left[\left(\beta_{1}\left(f_{1}\right), \ldots, \beta_{k}\left(f_{k}\right), \ldots\right)\right]
$$

for all $\left[\left(f_{1}, \ldots, f_{k}, \ldots\right)\right] \in \mathcal{S}^{\infty}(B)$.

Theorem 2.7 If $X$ is a Banach space with Property $(H)$, then the homomorphism $\beta$ induces an isomorphism

$$
\beta_{*}: K_{*}\left(\mathcal{S}^{\infty}(B)\right) \rightarrow K_{*}\left(\mathcal{A}^{\infty}(X, B)\right) .
$$

Proof For each $k$, endow $V_{k}$ with a Euclidean metric and let $D_{k}$ be the corresponding Dirac operator on $V_{k}$. Let $\alpha_{k}$ be the asymptotic morphism from $\mathcal{A}\left(V_{k}\right)$ to $\mathcal{S} \hat{\otimes} \mathcal{K}$ associated to $D_{k}$ :

$$
\alpha_{k}(t): f \hat{\otimes} h \rightarrow f\left(t^{-1}\left(s \hat{\otimes} 1+1 \hat{\otimes} D_{k}\right)\right) \pi(h)
$$


for all $f \in \mathcal{S}$ and $h \in \mathcal{C}\left(V_{k}\right)$, where $\mathcal{C}\left(V_{k}\right)$ is as in the definition of $\mathcal{A}\left(V_{k}\right), W_{k}$ (in the definition of $\left.\mathcal{C}\left(V_{k}\right)\right)$ is identified with $V_{k}$ as Euclidean vector spaces, and $\pi(h)$ is the multiplication operator associated to $h . \alpha_{k}$ induces an asymptotic morphism (still called $\left.\alpha_{k}\right)$ from $\mathcal{A}\left(V_{k}\right) \hat{\otimes} B \hat{\otimes} \mathcal{K}$ to $\mathcal{S} \hat{\otimes} B \hat{\otimes} \mathcal{K}$. This asymptotic morphism was first introduced by Higson-Kasparov-Trout [8].

For each $t \in[1, \infty)$, we define a map

$$
\alpha(t): \mathcal{A}^{\infty}(X, B) \rightarrow \mathcal{S}^{\infty}(B)
$$

by

$$
(\alpha(t))\left(\left[\left(a_{1}, \ldots, a_{k}, \ldots\right)\right]\right)=\left[\left(\left(\alpha_{1}(t)\right)\left(a_{1}\right), \ldots,\left(\alpha_{k}(t)\right)\left(a_{k}\right), \ldots\right)\right] .
$$

After rescaling $\alpha_{k}(t)$ for each $k, \alpha$ is an asymptotic morphism from $\mathcal{A}^{\infty}(X, B)$ to $\mathcal{S}^{\infty}(B)$, where the rescaling constant for each $k$ can be chosen as the Lipschitz constant of the Lipschitz equivalence between the Euclidean norm on $V_{k}$ and the Banach norm on $V_{k} \cdot \alpha$ is called the Dirac morphism. More precisely, let $C_{k}$ be the Lipschitz constant of the Lipschitz equivalence between the Euclidean norm $\|\cdot\|_{E}$ on $V_{k}$ and the Banach norm on $\|\cdot\|$ on $V_{k}$, that is, $C_{k}$ is a positive constant satisfying $C_{k}^{-1}\|v\|_{E} \leq\|v\| \leq C_{k}\|v\|_{E}$ for all $v \in V_{k}$. For each $k$, the scaled asymptotic morphism is defined to be $\alpha_{k}\left(C_{k} t\right)$ (still denoted by $\alpha_{k}$ ).

Let $\mathcal{A}^{\infty}(X \times X, B)$ be defined as $\mathcal{A}^{\infty}(X, B)$ using the sequence of finite dimensional subspaces $V_{n} \times V_{n}$ and $W_{n} \times W_{n}$.

We define a homomorphism $\beta_{k}^{\prime}$ from $\mathcal{A}\left(V_{k}\right)$ to $\mathcal{A}\left(V_{k} \times V_{k}\right)$ by

$$
\left(\beta_{k}^{\prime}(f \hat{\otimes} g)\right)\left(s, v_{k}, v_{k}^{\prime}\right)=f\left(\left(s, \phi\left(v_{k}^{\prime}\right)\right)\right) \hat{\otimes} g\left(v_{k}\right),
$$

where $f \in \mathcal{S}, g \in C_{0}\left(V_{k}, \operatorname{Clifford}\left(W_{k}\right)\right), s \in \mathbb{R},\left(v_{k}, v_{k}^{\prime}\right) \in V_{k} \times V_{k}$, and $f\left(\left(s, \phi\left(v_{k}^{\prime}\right)\right)\right)$ is defined to be $f\left(s \hat{\otimes} 1+1 \hat{\otimes} \phi\left(v_{k}^{\prime}\right)\right)$.

The homomorphism $\beta_{k}^{\prime}$ induces a homomorphism (which we also denote $\beta_{k}^{\prime}$ ) from $\mathcal{A}\left(V_{k}\right) \hat{\otimes} B \hat{\otimes} \mathcal{K}$ to $\mathcal{A}\left(V_{k} \times V_{k}\right) \hat{\otimes} B \hat{\otimes} \mathcal{K}$. Using $\left\{\beta_{k}^{\prime}\right\}$ we can define a homomorphism $\beta^{\prime}$ from $\mathcal{A}^{\infty}(X, B)$ to $\mathcal{A}^{\infty}(X \times X, B)$. Similarly we define the Dirac morphism $\alpha^{\prime}$ from $\mathcal{A}^{\infty}(X \times X, B)$ to $\mathcal{A}^{\infty}(X, B)$ using the Dirac operator on the first copy of $V_{k}$ in $V_{k} \times V_{k}$ for each $k$.

We now apply Atiyah's rotation trick [1]. For any $\theta \in\left[0, \frac{\pi}{2}\right]$, we define the rotation $R_{\theta}$ by

$$
R_{\theta}(v, w)=(\cos \theta v-\sin \theta w, \sin \theta v+\cos \theta w) .
$$


This rotation $R_{\theta}$ induces an automorphism (also denoted $\left.R_{\theta}\right)$ on $\mathcal{A}^{\infty}(X \times X, B)$. In particular, for any sequence of vectors $\left\{\left(c_{k}, c_{k}^{\prime}\right)\right\}$ such that $\left(c_{k}, c_{k}^{\prime}\right) \in V_{k} \times V_{k}$, we have

$$
\begin{aligned}
& R_{\theta}:\left[\left(f_{k}\left(\left(s,\left(\phi\left(v_{k}-c_{k}\right), \phi\left(v_{k}^{\prime}-c_{k}^{\prime}\right)\right)\right)\right)\right)\right] \rightarrow \\
& \quad\left[\left(f _ { k } \left(\left(s,\left(\cos \theta \phi\left(\cos \theta v_{k}+\sin \theta v_{k}^{\prime}-c_{k}\right)-\sin \theta \phi\left(-\sin \theta v_{k}+\cos \theta v_{k}^{\prime}-c_{k}^{\prime}\right),\right.\right.\right.\right.\right. \\
& \left.\left.\left.\left.\left.\quad \sin \theta \phi\left(\cos \theta v_{k}+\sin \theta v_{k}^{\prime}-c_{k}\right)+\cos \theta \phi\left(-\sin \theta v_{k}+\cos \theta v_{k}^{\prime}-c_{k}^{\prime}\right)\right)\right)\right)\right)\right],
\end{aligned}
$$

where $f_{k} \in \mathcal{S},\left\{f_{k}\right\}$ is equicontinuous, and $s \in \mathbb{R},\left(v_{k}, v_{k}^{\prime}\right) \in V_{k} \times V_{k}$.

By homotopy invariance, we have

$$
(\mathrm{Id})_{*}=\left(R_{0}\right)_{*}=\left(R_{\frac{\pi}{2}}\right)_{*},
$$

where $\left(R_{\theta}\right)_{*}$ is the automorphism on $K_{*}\left(\mathcal{A}^{\infty}(X \times X, B)\right)$ induced by $R_{\theta}$.

For any $x \in K_{*}\left(\mathcal{S}^{\infty}(B)\right)$, we have

$$
\alpha_{*}\left(\beta_{*}(x)\right)=x .
$$

For any $y \in K_{*}\left(\mathcal{A}^{\infty}(X, B)\right)$, we have

$$
\beta_{*}\left(\alpha_{*}(y)\right)=\alpha_{*}^{\prime}\left(\beta_{*}^{\prime}(y)\right)=\alpha_{*}^{\prime}\left(R_{\frac{\pi}{2}}\right)_{*}\left(\beta_{*}^{\prime}(y)\right)=\tilde{y},
$$

where the map $y \rightarrow \tilde{y}$ is induced by the map $\left(v_{k}\right) \rightarrow\left(-v_{k}\right)$.

The above two identities imply that $\beta$ and $\alpha$ are isomorphisms, inverse to each other, and $y \rightarrow \tilde{y}$ is the identity map.

The following result follows from Theorem 2.7 and the Green-Julg theorem.

Corollary 2.8 Let $H$ be a finite group and let $B$ be a graded $H-C^{*}$-algebra. If $X$ is a Banach space with Property $(H)$, then $\beta$ induces an isomorphism

$$
\beta_{*}: K_{*}^{H}\left(\mathcal{S}^{\infty}(B)\right) \rightarrow K_{*}^{H}\left(\mathcal{A}^{\infty}(X, B)\right) .
$$

Proposition 2.9 If $X$ is a Banach space with rational Property $(H)$, then the homomorphism $\beta$ induces an isomorphism

$$
\beta_{*}: K_{*}\left(\mathcal{S}^{\infty}(B)\right) \otimes \mathbb{Q} \rightarrow K_{*}\left(\mathcal{A}^{\infty}(X, B)\right) \otimes \mathbb{Q} .
$$

Corollary 2.10 Let $H$ be a finite group and let $B$ be a graded $H-C^{*}$-algebra. If $X$ is a Banach space with rational Property $(H)$, then $\beta$ induces an isomorphism

$$
\beta_{*}: K_{*}^{H}\left(\mathcal{S}^{\infty}(B)\right) \otimes \mathbb{Q} \rightarrow K_{*}^{H}\left(\mathcal{A}^{\infty}(X, B)\right) \otimes \mathbb{Q} .
$$




\section{The Baum-Connes map and localization}

In this section, we briefly recall the Baum-Connes map and its relation to the localization algebra. Our reformulation of the Baum-Connes map follows the work of Roe [12] and uses a localization technique introduced by $\mathrm{Yu}$ [14]. This reformulation will be useful in the next section.

Let $\Gamma$ be a countable discrete group. Let $\Delta$ be a locally compact metric space with a proper and cocompact isometric action of $\Gamma$. Let $C_{0}(\Delta)$ be the algebra of all complex valued continuous functions on $\Delta$ which vanish at infinity. Let $B$ be a $\Gamma-C^{*}$-algebra.

The following definition is due to John Roe [12].

Definition 3.1 Let $H$ be a Hilbert module over $B$ and let $\varphi$ be a $*$-homomorphism from $C_{0}(\Delta)$ to $B(H)$, the $C^{*}$-algebra of all bounded (adjointable) operators on $H$. Let $T$ be an operator in $B(H)$.

(1) The support of $T$ is defined to be the complement (in $\Delta \times \Delta$ ) of the set of all points $(x, y) \in \Delta \times \Delta$ for which there exists $f \in C_{0}(\Delta)$ and $g \in C_{0}(\Delta)$ satisfying $\varphi(f) T \varphi(g)=0$ and $f(x) \neq 0$ and $g(y) \neq 0$;

(2) The propagation of $T$ is defined to be $\sup \{d(x, y):(x, y) \in \operatorname{Supp}(T)\}$;

(3) $T$ is said to be locally compact if $\varphi(f) T$ and $T \varphi(f)$ are in $K(H)$ for all $f \in C_{0}(\Delta)$, where $K(H)$ is defined to be the operator norm closure of all finite rank operators on the Hilbert module $H$.

Let $H$ be a (countably generated) $\Gamma$-Hilbert module over $B$ and let $\varphi$ be a $*-$ homomorphism from $C_{0}(\Delta)$ to $B(H)$ which is covariant in the sense that $\varphi(\gamma f) h=$ $\left(\gamma(\varphi(f)) \gamma^{-1}\right) h$ for all $\gamma \in \Gamma, f \in C_{0}(\Delta)$ and $h \in H$. Such a triple $\left(C_{0}(\Delta), \Gamma, \varphi\right)$ is called a covariant system.

Definition 3.2 We define the covariant system $\left(C_{0}(\Delta), \Gamma, \varphi\right)$ to be admissible if

(1) the $\Gamma$-action on $\Delta$ is proper and cocompact;

(2) there exist a $\Gamma$-Hilbert space $H_{\Delta}$ and a separable and infinite dimensional $\Gamma$-Hilbert space $E$ such that

(a) $H$ is isomorphic to $H_{\Delta} \otimes E \otimes B$ as $\Gamma$-Hilbert modules over $B$;

(b) $\varphi=\varphi_{0} \otimes I$ for some $\Gamma$-equivariant $*$-homomorphism $\varphi_{0}$ from $C_{0}(\Delta)$ to $B\left(H_{\Delta}\right)$ such that $\varphi_{0}(f)$ is not in $K\left(H_{\Delta}\right)$ for any nonzero function $f \in$ $C_{0}(\Delta)$ and $\varphi_{0}$ is nondegenerate in the sense that $\left\{\varphi_{0}(f) H_{\Delta}: f \in C_{0}(\Delta)\right\}$ is dense in $H_{\Delta}$, 
(c) for each $x \in \Delta, E$ is isomorphic to $l^{2}\left(\Gamma_{x}\right) \otimes H_{x}$ as $\Gamma_{x}$-Hilbert spaces for some Hilbert space $H_{x}$ with a trivial $\Gamma_{x}$ action, where $\Gamma_{x}$ is the finite isotropy subgroup of $\Gamma$ at $x$.

In the above definition, the $\Gamma_{x}$-action on $l^{2}\left(\Gamma_{x}\right)$ is regular, that is, $(\gamma \xi)(z)=\xi\left(\gamma^{-1} z\right)$ for every $\gamma \in \Gamma_{x}, \xi \in l^{2}\left(\Gamma_{x}\right)$, and $z \in \Gamma_{x}, B$ is the $\Gamma$-Hilbert module over $B$ with the inner product $\langle a, b\rangle=a^{*} b$, and $I$ is the identity operator on $E \otimes B$.

We remark that such an admissible covariant system always exists (for example we can choose $E$ to be $l^{2}(\Gamma)$ with a regular action $\Gamma$ ). We point out that condition (2) implies that $E$ contains all unitary representations of the finite isotropy groups and this point is important for Proposition 3.4 of this section.

Definition 3.3 Let $\left(C_{0}(\Delta), \Gamma, \varphi\right)$ be an admissible covariant system. We define $\mathbb{C}(\Gamma, \Delta, B)$ to be the algebra of $\Gamma$-invariant locally compact operators in $B(H)$ with finite propagation. The $C^{*}$-algebra $C_{r}^{*}(\Gamma, \Delta, B)$ is the operator norm closure of $\mathbb{C}(\Gamma, \Delta, B)$.

The following result is essentially due to John Roe.

Proposition 3.4 If $\left(C_{0}(\Delta), \Gamma, \varphi\right)$ is an admissible covariant system then $C_{r}^{*}(\Gamma, \Delta, B)$ is $*$-isomorphic to $\left(B \rtimes_{r} \Gamma\right) \otimes \mathcal{K}$, where $B \rtimes_{r} \Gamma$ is the reduced crossed product $C^{*}$ algebra and $\mathcal{K}$ is the algebra of all compact operators on a separable and infinite dimensional Hilbert space.

Proof Proposition 3.4 follows from the definitions of the admissible covariant system, $C_{r}^{*}(\Gamma, \Delta, B)$, and the reduced crossed product $C^{*}$-algebra.

Next we will describe the Baum-Connes map.

Let $H$ be a $\Gamma$-Hilbert module over $B$, let $F$ be an operator in $B(H)$, let $\varphi$ be a $*$-homomorphism from $C_{0}(\Delta)$ to $B(H)$ such that $F$ is $\Gamma$-equivariant, that is, $\gamma F \gamma^{-1}=F$ for all $\gamma \in \Gamma, \varphi(f) F-F \varphi(f), \varphi(f)\left(F F^{*}-I\right)$ and $\varphi(f)\left(F^{*} F-I\right)$ are in $K(H)$ for all $f \in C_{0}(\Delta)$.

We denote the group $K K_{0}^{\Gamma}\left(C_{0}(\Delta), B\right)$ by $K K_{0}^{\Gamma}(\Delta, B) .(H, \varphi, F)$ gives a $K K$-cycle representing a class in $K K_{0}^{\Gamma}(\Delta, B)$. It is not difficult to prove that every class in $K K_{0}^{\Gamma}(\Delta, B)$ is equivalent to $(H, \varphi, F)$ such that $\left(C_{0}(\Delta), \Gamma, \varphi\right)$ is an admissible covariant system. This can be seen as follows. We define a new $K K$-group $\widetilde{K K}_{*}^{\Gamma}(\Delta, B)$ using $K K$-cycles $(H, \varphi, F)$ such that $\left(C_{0}(\Delta), \Gamma, \varphi\right)$ is an admissible covariant system. By the proof of [10, Proposition 5.5] by Kasparov and Skandalis, we can show that 
there exists a $\Gamma$-Hilbert space $H_{\Delta}$ satisfying the conditions of Definition 3.2 such that $H \oplus\left(H_{\Delta} \otimes l^{2}(\Gamma) \otimes B\right)$ is isomorphic to $H_{\Delta} \otimes l^{2}(\Gamma) \otimes B$ as $\Gamma$-Hilbert modules over $B$. Using this stabilization result, we can prove that the natural homomorphism from $\widetilde{K K}_{*}^{\Gamma}(\Delta, B)$ to $K K_{0}^{\Gamma}(\Delta, B)$ is an isomorphism.

For any $\epsilon>0$, let $\left\{U_{i}\right\}_{i \in I}$ be a locally finite and $\Gamma$-equivariant open cover of $\Delta$ satisfying diameter $\left(U_{i}\right)<\epsilon$ for all $i$. Let $\left\{\psi_{i}\right\}$ be a $\Gamma$-equivariant partition of unity subordinate to $\left\{U_{i}\right\}_{i \in I}$. We define

$$
F_{\epsilon}=\sum_{i \in I} \varphi\left(\sqrt{\psi_{i}}\right) F \varphi\left(\sqrt{\psi_{i}}\right)
$$

where the convergence is in the strict topology.

Note that $F_{\epsilon}$ has propagation $\epsilon$ and $\left(H, \varphi, F_{\epsilon}\right)$ is equivalent to $(H, \varphi, F)$ in $K K_{0}^{\Gamma}(\Delta, B)$. Also $F_{\epsilon}$ is a multiplier of $C_{r}^{*}(\Gamma, \Delta, B)$, and is invertible modulo $C_{r}^{*}(\Gamma, \Delta, B)$.

Let $\partial$ be the boundary map

$$
K_{1}\left(M\left(C_{r}^{*}(\Gamma, \Delta, B)\right) / C_{r}^{*}(\Gamma, \Delta, B)\right) \rightarrow K_{0}\left(C_{r}^{*}(\Gamma, \Delta, B)\right)
$$

in $K$-theory, where $M\left(C_{r}^{*}(\Gamma, \Delta, H)\right)$ is the multiplier algebra of $C_{r}^{*}(\Gamma, \Delta, H)$. We can define the Baum-Connes map

$$
\mu: K K_{0}^{\Gamma}(\Delta, B) \rightarrow K_{0}\left(C_{r}^{*}(\Gamma, \Delta, B)\right) \cong K_{0}\left(B \rtimes_{r} \Gamma\right)
$$

by

$$
\mu([(H, \varphi, F)])=\partial\left(\left[F_{\epsilon}\right]\right) .
$$

More precisely the Baum-Connes map can be implemented as follows.

Let $p_{\epsilon}$ be the idempotent

$$
\left(\begin{array}{cc}
F_{\epsilon} F_{\epsilon}^{*}+\left(I-F_{\epsilon} F_{\epsilon}^{*}\right) F_{\epsilon} F_{\epsilon}^{*} & F_{\epsilon}\left(I-F_{\epsilon}^{*} F_{\epsilon}\right)+\left(I-F_{\epsilon} F_{\epsilon}^{*}\right) F_{\epsilon}\left(I-F_{\epsilon}^{*} F_{\epsilon}\right) \\
\left(I-F_{\epsilon}^{*} F_{\epsilon}\right) F_{\epsilon}^{*} & \left(I-F_{\epsilon}^{*} F_{\epsilon}\right)^{2}
\end{array}\right) .
$$

Observe that the propagation of $p_{\epsilon}$ is at most $5 \epsilon$.

Let

$$
p_{0}=\left(\begin{array}{ll}
I & 0 \\
0 & 0
\end{array}\right)
$$

We have

$$
\mu([(H, \varphi, F)])=\left[p_{\epsilon}\right]-\left[p_{0}\right] .
$$

Similarly we can define the Baum-Connes map

$$
\mu: K K_{1}^{\Gamma}(\Delta, B) \rightarrow K_{1}\left(C_{r}^{*}(\Gamma, \Delta, B)\right) \cong K_{1}\left(B \rtimes_{r} \Gamma\right) .
$$


This induces the Baum-Connes map

$$
\mu: K K_{*}^{\Gamma}(\mathcal{E} \Gamma, B) \rightarrow K_{*}\left(B \rtimes_{r} \Gamma\right),
$$

where $K K_{*}^{\Gamma}(\mathcal{E} \Gamma, B)$ is defined to be the inductive limit of $K K_{*}^{\Gamma}(\Delta, B)$ over all $\Gamma-$ invariant and cocompact subspaces $\Delta$ of $\mathcal{E} \Gamma$ which are finite dimensional simplicial polyhedra. Here we choose a model of $\mathcal{E} \Gamma$ so that $\mathcal{E} \Gamma$ is equal to the union of $\Gamma-$ invariant and cocompact subspaces $\Delta$ of $\mathcal{E} \Gamma$ which are finite dimensional simplicial polyhedra (the existence of such model follows from the construction of $\mathcal{E} \Gamma$ in the proof of [2, Proposition 1.7] by Baum, Connes and Higson, which is based on Milnor's join construction).

Let $\Delta$ be a locally compact and finite dimensional simplicial polyhedron. We endow $\Delta$ with the simplicial metric. Let $\left(C_{0}(\Delta), \Gamma, \varphi\right)$ be an admissible covariant system as before, where $\varphi$ is a $*$-homomorphism from $C_{0}(\Delta)$ to $B(H)$ for some Hilbert module $H$ over $B$.

\section{Definition 3.5}

(1) The algebraic localization algebra $\mathbb{C}_{L}(\Gamma, \Delta, B)$ is defined to be the algebra of all bounded and uniformly continuous functions $f:[0, \infty) \rightarrow \mathbb{C}(\Gamma, \Delta, B)$ such that the propagation of $f(t)$ goes to 0 as $t \rightarrow \infty$, where $\mathbb{C}(\Gamma, \Delta, B)$ is as in Definition 3.3.

(2) The localization algebra $C_{L}^{*}(\Gamma, \Delta, B)$ is the norm closure of $\mathbb{C}_{L}(\Gamma, \Delta, B)$ with respect to the norm

$$
\|f\|=\sup _{t \in[0, \infty)}\|f(t)\| .
$$

It is not difficult to prove that, up to a $*$-isomorphism, $\mathbb{C}_{L}(\Gamma, \Delta, B)$ and $C_{L}^{*}(\Gamma, \Delta, B)$ are independent of the choices made in Definition 3.2. The localization algebra is an equivariant analogue of the algebra introduced by $\mathrm{Yu}$ in [14].

Any class in $K K_{0}^{\Gamma}(\Delta, B)$ can be represented by $(H, \varphi, F)$ such that the covariant system $\left(C_{0}(\Delta), \Gamma, \varphi\right)$ is admissible, where $H$ is a $\Gamma$-Hilbert module over $B, F$ is an operator in $B(H), \varphi$ is a $*$-homomorphism from $C_{0}(\Delta)$ to $B(H)$ such that $F$ is $\Gamma$-equivariant, and $\varphi(f) F-F \varphi(f), \varphi(f)\left(F F^{*}-I\right)$ and $\varphi(f)\left(F^{*} F-I\right)$ are in $K(H)$ for all $f \in C_{0}(\Delta)$.

For each natural number $n$, we let $F_{\frac{1}{n}}$ be defined as above. We define an operator valued function $F(t)$ on $[0, \infty)$ by

$$
F(t)=(t-n+1) F_{\frac{1}{n}}+(t-n) F_{\frac{1}{n+1}}
$$


for all $t \in[n, n+1]$.

$F(t)$ is a multiplier of $C_{L}^{*}(\Gamma, \Delta, B)$ and is invertible modulo $C_{L}^{*}(\Gamma, \Delta, B)$. We define the local Baum-Connes map

$$
\mu_{L}: K K_{0}^{\Gamma}(\Delta, B) \rightarrow K_{0}\left(C_{L}^{*}(\Gamma, \Delta, B)\right),
$$

by

$$
\left.\mu_{L}[H, \varphi, F)\right]=\partial[F(t)]
$$

where

$$
\text { ว: } K_{1}\left(M\left(C_{L}^{*}(\Gamma, \Delta, B)\right) / C_{L}^{*}(\Gamma, \Delta, B)\right) \rightarrow K_{0}^{*}\left(C_{L}^{*}(\Gamma, \Delta, B)\right),
$$

is the boundary map in $K$-theory and $M\left(C_{L}^{*}(\Gamma, \Delta, B)\right)$ is the multiplier algebra of $C_{L}^{*}(\Gamma, \Delta, B)$.

Similarly we can define the local Baum-Connes map

$$
\mu_{L}: K K_{1}^{\Gamma}(\Delta, B) \rightarrow K_{1}\left(C_{L}^{*}(\Gamma, \Delta, B)\right) .
$$

We remark that the local Baum-Connes map is very much in the spirit of the local index theory of elliptic differential operators.

Theorem 3.6 Let $B$ be a $\Gamma-C^{*}$-algebra. The local Baum-Connes map $\mu_{L}$ is an isomorphism from $K K_{*}^{\Gamma}(\Delta, B)$ to $K_{*}\left(C_{L}^{*}(\Gamma, \Delta, B)\right)$ if $\Delta$ is a finite dimensional simplicial polyhedron with the $\Gamma$-invariant simplicial metric.

Proof This theorem is a consequence of the Mayer-Vietoris and five lemma argument (essentially similar to the proof of the non-equivariant analogue of $\mathrm{Yu}$ [14]).

Let $C_{L}^{*}(\Gamma, \mathcal{E} \Gamma, B)$ be the $C^{*}$-algebra inductive limit of $C_{L}^{*}(\Gamma, \Delta, B)$, where the limit is taken over all $\Gamma$-invariant and cocompact subspaces $\Delta$ of $\mathcal{E} \Gamma$ which are finite dimensional simplicial polyhedra.

The above local Baum-Connes map induces a map (still called the local Baum-Connes map)

$$
\mu_{L}: K K_{*}^{\Gamma}(\mathcal{E} \Gamma, B) \rightarrow K_{*}\left(C_{L}^{*}(\Gamma, \mathcal{E} \Gamma, B)\right) .
$$

Corollary 3.7 The local Baum-Connes map $\mu_{L}$ is an isomorphism from $K K_{*}^{\Gamma}(\mathcal{E} \Gamma, B)$ to $K_{*}\left(C_{L}^{*}(\Gamma, \mathcal{E} \Gamma, B)\right)$.

Next we shall discuss the relation between the Baum-Connes map and an evaluation map. This connection will be useful in the proof of the main theorem in Section 5. 
Let $e$ be the evaluation map

$$
C_{L}^{*}(\Gamma, \Delta, B) \rightarrow C_{r}^{*}(\Gamma, \Delta, B) \cong\left(B \rtimes_{r} \Gamma\right) \otimes \mathcal{K}
$$

defined by

$$
e(f)=f(0)
$$

for all $f \in C_{L}^{*}(\Gamma, \Delta, B)$.

The above evaluation maps induce an evaluation homomorphism (still denoted by $e$ )

$$
C_{L}^{*}(\Gamma, \mathcal{E} \Gamma, B) \rightarrow\left(B \rtimes_{r} \Gamma\right) \otimes \mathcal{K} .
$$

We have

$$
\mu=e_{*} \circ \mu_{L} .
$$

\section{The Bott map}

In this section, we introduce a Bott map for $K$-groups. The Bott map will play an essential role in the proof of the main result of this paper.

Let $X$ be a Banach space with (rational) Property (H). Let $\Gamma$ be a countable group with a left invariant proper length metric. Let $h: \Gamma \rightarrow X$ be a coarse embedding. Without loss of generality we can assume that the image of $h$ is contained in $V$, where $V$ is as in the definition of the (rational) Property $(\mathrm{H})$.

For each $v \in V, w \in W$, and $\gamma \in \Gamma$, we define bounded functions $\xi_{v, \gamma}$ and $\eta_{v, w, \gamma}$ on $\Gamma$ by

$$
\begin{aligned}
\xi_{v, \gamma}(x) & =\|\phi(v+h(x)-h(x \gamma))\| \\
& =\|v+h(x)-h(x \gamma)\|, \\
\eta_{v, w, \gamma}(x) & =\langle\phi(v+h(x)-h(x \gamma)), w\rangle
\end{aligned}
$$

for all $x \in \Gamma$, where $\phi$ is defined as in Section 2. The boundedness of $\xi_{v, \gamma}$ and $\eta_{v, w, \gamma}$ follows from the fact that $h$ is a coarse embedding.

Let $c_{0}(\Gamma)$ be the algebra of all functions on $\Gamma$ vanishing at infinity. We define the $\Gamma$ action on $l^{\infty}(\Gamma)$ by $(\gamma(\eta))(x)=\eta(x \gamma)$ for each $\eta \in l^{\infty}(\Gamma), \gamma \in \Gamma, x \in \Gamma$. Let $Y$ be the spectrum of the unital commutative $\Gamma$-invariant $C^{*}$-subalgebra of $l^{\infty}(\Gamma)$ generated by $c_{0}(\Gamma)$, all constant functions on $\Gamma$, all $\xi_{v, \gamma}$ and $\eta_{v, w, \gamma}$, and their translations by group elements of $\Gamma$. Notice that $Y$ is a separable compact space and is a quotient space of $\beta \Gamma$, the Stone-Čech compactification of $\Gamma$. 
Let $\mathcal{A}(X)$ be the $C^{*}$-algebra associated to $X$ (as defined in Section 2). If $A$ is a $\Gamma-C^{*}$-algebra, then $\Gamma$ acts on the $C^{*}$-algebra

$$
C(Y) \hat{\otimes} \mathcal{A}(X) \hat{\otimes} A \subseteq l^{\infty}(\Gamma) \hat{\otimes} \mathcal{A}(X) \hat{\otimes} A
$$

by

$\gamma\left(\eta(x) \hat{\otimes} f\left(\left(s, \phi\left(v-v_{0}\right)\right)\right) \hat{\otimes} a\right)=\eta(x \gamma) \hat{\otimes} f\left(\left(s, \phi\left(v-v_{0}+h(x)-h(x \gamma)\right)\right) \hat{\otimes} \gamma(a)\right.$

for each $\gamma \in \Gamma, \eta \in C(Y) \subseteq l^{\infty}(\Gamma), x \in \Gamma, s \in \mathbb{R}, f \in C_{0}(\mathbb{R}), v \in V, v_{0} \in V$ and $a \in A$, where $f\left(\left(s, \phi\left(v-v_{0}\right)\right)\right)$ is as in the definition of $\mathcal{A}(X)$.

The above $\Gamma$ action on $C(Y) \hat{\otimes} \mathcal{A}(X) \hat{\otimes} A$ has its origin in work by Yu [15] and Skandalis, Tu and Yu [13]. We can see that the $\Gamma$ action on $C(Y) \hat{\otimes} \mathcal{A}(X) \hat{\otimes} A$ is well defined as follows. Recall that, for any net $\left\{w_{i}\right\}_{i \in I}$ in a Hilbert space, if $w_{i}$ converges to a vector $w$ of the Hilbert space in weak topology and $\left\|w_{i}\right\|$ converges to $\|w\|$, then $w_{i}$ converges to $w$ in norm. Using this fact and the definition of $Y$, we know that $\phi\left(v-v_{0}+h(x)-h(x \gamma)\right)$ can be extended to a norm continuous function on $Y$ with values in $H$. It follows that $f\left(\left(s, \phi\left(v-v_{0}+h(x)-h(x \gamma)\right)\right)\right.$ can be identified with a norm continuous function on $Y$ with values in $\mathcal{A}(X) \hat{\otimes} A$. This, together with the compactness of $Y$, implies that $\gamma\left(\eta(x) \hat{\otimes} f\left(\left(s, \phi\left(v-v_{0}\right)\right)\right) \hat{\otimes} a\right)$ is an element in $C(Y) \hat{\otimes} \mathcal{A}(X) \hat{\otimes} A$. Let $\mathcal{D}$ be the subalgebra of $C(Y) \hat{\otimes} \mathcal{A}(X) \hat{\otimes} A$ consisting of all linear combinations of products of elements of the type $\eta(x) \hat{\otimes} f\left(\left(s, \phi\left(v-v_{0}\right)\right)\right) \hat{\otimes} a$. Note that, by the definition of $\mathcal{A}(X), \mathcal{D}$ is dense in the $C^{*}$-algebra $C(Y) \hat{\otimes} \mathcal{A}(X) \hat{\otimes} A$. By extending linearly and multiplicatively, for each $d \in \mathcal{D}$, we can define $\gamma(d)$ as an element of $C(Y) \hat{\otimes} \mathcal{A}(X) \hat{\otimes} A$. By the definition of $Y, \Gamma$ is a dense subset of $Y$. This, together with the definition of $\gamma(d)$, implies that $\|\gamma(d)\|=\|d\|$ for each $d \in \mathcal{D}$. Finally the $\Gamma$ action extends continuously to $C(Y) \hat{\otimes} \mathcal{A}(X) \hat{\otimes} A$.

Let $Z$ be the space of all probability measures on $Y$ with the weak topology. Note that $Z$ is a convex and compact topological space with the weak* topology. The idea of using the space of probability measures is due to Nigel Higson [7]. The action of $\Gamma$ on $C(Y) \hat{\otimes} \mathcal{A}(X) \otimes A$ induces an action of $\Gamma$ on $C(Z) \hat{\otimes} \mathcal{A}(X) \hat{\otimes} A$ by

$\gamma\left(u(\mu) \hat{\otimes} f\left(\left(s, \phi\left(v-v_{0}\right)\right)\right) \hat{\otimes} a\right)=$

$$
u(\gamma(\mu)) \hat{\otimes} f\left(\left(s, \phi\left(v-v_{0}+\int_{Y}(h(y)-h(y \gamma)) d \mu\right)\right)\right) \hat{\otimes} \gamma(a)
$$

for each $\gamma \in \Gamma, u \in C(Z), \mu \in Z, f \in C_{0}(\mathbb{R}), s \in \mathbb{R}, v \in V, v_{0} \in V$ and $a \in A$, where the $\Gamma$ action on $Z$ is induced by the $\Gamma$ action on $Y$. The assumption that $h$ is a coarse embedding implies that $C(Z) \hat{\otimes} \mathcal{A}(X) \hat{\otimes} A$ is a $\Gamma$-proper $C^{*}$-algebra. This can be seen as follows: let $\mathcal{C}(X)$ be the abelian $C^{*}$-subalgebra of $C(Z) \hat{\otimes} \mathcal{A}(X)$ generated by all elements $b \hat{\otimes} g\left(s^{2}+\left\|v-v_{0}\right\|^{2}\right)$, where $b \in C(Z), g \in C_{0}(\mathbb{R})$ and $v_{0} \in V . \mathcal{C}(X)$ 
is isomorphic to a commutative $C^{*}$-algebra $C_{0}(T)$ for some locally compact space $T$. Notice that $T$ is a proper $\Gamma$-space (as a consequence of the fact that $h$ is a coarse embedding), $\mathcal{C}(X)$ is in the center of the multiplier algebra of $C(Z) \hat{\otimes} \mathcal{A}(X) \hat{\otimes} A$, and $\mathcal{C}(X)(C(Z) \hat{\otimes} \mathcal{A}(X) \hat{\otimes} A)$ is dense in $C(Z) \hat{\otimes} \mathcal{A}(X) \hat{\otimes} A$.

For each $f \in \mathcal{S}$, let $f_{t} \in \mathcal{A}(X)$ be defined by

$$
f_{t}((s, v))=f\left(t^{-1}(s, \phi(v))\right)
$$

for all $s \in \mathbb{R}, v \in V$.

We define the Bott map

$$
\beta: K_{*}\left((C(Z) \hat{\otimes} \mathcal{S} \hat{\otimes} A) \rtimes_{r} \Gamma\right) \rightarrow K_{*}\left((C(Z) \hat{\otimes} \mathcal{A}(X) \hat{\otimes} A) \rtimes_{r} \Gamma\right)
$$

to be the homomorphism induced by the asymptotic morphism

$$
\beta_{t}((u \hat{\otimes} f \hat{\otimes} a) \gamma)=\left(u \hat{\otimes} f_{t} \hat{\otimes} a\right) \gamma
$$

from $(C(Z) \hat{\otimes} \mathcal{S} \hat{\otimes} A) \rtimes_{r} \Gamma$ to $(C(Z) \hat{\otimes} \mathcal{A}(X) \hat{\otimes} A) \rtimes_{r} \Gamma$, for $t \in[1, \infty), u \in C(Z)$, $f \in \mathcal{S}, a \in A$ and $\gamma \in \Gamma$.

The fact that $\beta_{t}$ is an asymptotic morphism follows from the identity $\phi(c v)=|c| \phi(v)$ for any scalar $c$ and the assumption that the restriction of $\phi$ to the sphere of $V$ is uniformly continuous.

Let $\Delta$ be a locally compact metric space with a proper and cocompact isometric action of $\Gamma$. We define an asymptotic morphism

$$
\beta_{t}: C_{L}^{*}(\Gamma, \Delta, C(Z) \hat{\otimes} \mathcal{S} \hat{\otimes} A) \rightarrow C_{L}^{*}(\Gamma, \Delta, C(Z) \hat{\otimes} \mathcal{A}(X) \hat{\otimes} A)
$$

induced by the homomorphism from $\mathcal{S}$ to $\mathcal{A}(X): f \rightarrow f_{t}$, where

$$
f_{t}((s, v))=f\left(t^{-1}(s, \phi(v))\right)
$$

for all $f \in \mathcal{S}$ and $s \in \mathbb{R}, v \in V$.

More precisely $\beta_{t}$ is defined as follows. Let $\left(C_{0}(\Delta), \Gamma, \varphi\right)$ be an admissible covariant system, where $\varphi$ is a $*$-homomorphism from $C_{0}(\Delta)$ to $B(H)$ for some Hilbert module $H$ over $C(Z) \hat{\otimes} \mathcal{S} \hat{\otimes} A$. By the definition of admissible covariant system, we have

$$
K(H) \cong C(Z) \hat{\otimes} \mathcal{S} \hat{\otimes} A \hat{\otimes} \mathcal{K},
$$

where $K(H)$ is the operator norm closure of all finite rank operators on the Hilbert module $H$ and $\mathcal{K}$ is the graded $C^{*}$-algebra of all compact operators on a graded separable and infinite dimensional Hilbert space. Let $\beta_{t}^{\prime}$ be the asymptotic morphism from $C(Z) \hat{\otimes} \mathcal{S} \hat{\otimes} A \hat{\otimes} \mathcal{K}$ to $C(Z) \hat{\otimes} \mathcal{A}(X) \hat{\otimes} A \hat{\otimes} \mathcal{K}$ induced by the homomorphism from $\mathcal{S}$ to $\mathcal{A}(X): f \rightarrow f_{t}$, where $f_{t}$ is defined as in the previous paragraph. 
Let $c$ be a $\Gamma$ cut-off function on $\Delta$, that is, $c$ is a compactly supported non-negative continuous function on $\Delta$ satisfying

$$
\sum_{\gamma \in \Gamma} c\left(\gamma^{-1} x\right)=1
$$

for all $x \in \Delta$. The existence of such cut-off function follows from properness and cocompactness of the $\Gamma$ action on $\Delta$.

For each $T \in \mathbb{C}_{L}(\Gamma, \Delta, C(Z) \hat{\otimes} \mathcal{S} \hat{\otimes} A)$, we define

$$
\beta_{t}(T)=\sum_{\gamma \in \Gamma} \beta_{t}^{\prime}\left(\varphi(\gamma(c)) \gamma T \gamma^{-1}\right)
$$

in $\mathbb{C}_{L}(\Gamma, \Delta, C(Z) \hat{\otimes} \mathcal{A}(X) \hat{\otimes} A)$, where $\beta_{t}^{\prime}$ is the asymptotic morphism defined above, $(\gamma(c))(x)=c\left(\gamma^{-1} x\right)$ for all $x \in \Delta$, and the sum converges in the strong operator topology because $T$ has finite propagation.

Note that $\beta_{t}(T)$ is $\Gamma$-invariant and is an element of $\mathbb{C}_{L}(\Gamma, \Delta, C(Z) \hat{\otimes} \mathcal{A}(X) \hat{\otimes} A)$. It is not difficult to see that $\beta_{t}$ can be extended to an asymptotic morphism

$$
\beta_{t}: C_{L}^{*}(\Gamma, \Delta, C(Z) \hat{\otimes} \mathcal{S} \hat{\otimes} A) \rightarrow C_{L}^{*}(\Gamma, \Delta, C(Z) \hat{\otimes} \mathcal{A}(X) \hat{\otimes} A) .
$$

By Corollary 3.7, the above asymptotic morphism induces a homomorphism

$$
\beta: K K_{*}^{\Gamma}(\mathcal{E} \Gamma, C(Z) \hat{\otimes} \mathcal{S} \hat{\otimes} A) \rightarrow K K_{*}^{\Gamma}(\mathcal{E} \Gamma, C(Z) \hat{\otimes} \mathcal{A}(X) \hat{\otimes} A),
$$

called the Bott map.

\section{Proposition 4.1 The Bott map}

$$
\beta: K K_{*}^{\Gamma}(\mathcal{E} \Gamma, C(Z) \hat{\otimes} \mathcal{S} \hat{\otimes} A) \rightarrow K K_{*}^{\Gamma}(\mathcal{E} \Gamma, C(Z) \hat{\otimes} \mathcal{A}(X) \hat{\otimes} A)
$$

is injective, where $\mathcal{E} \Gamma$ is the universal space for proper $\Gamma$-actions.

Proof Let $\mathcal{A}^{\infty}(X, A)$ be as defined in Section 2. For any fixed $v_{0} \in V_{k} \subseteq V$, we note that the translation

$$
\begin{aligned}
{\left[\left(f_{1}\left(s, v_{1}\right), \ldots, f_{k-1}\left(s, v_{k-1}\right)\right.\right.} & \left.\left., f_{k}\left(s, v_{k}\right), f_{k+1}\left(s, v_{k+1}\right), \ldots\right)\right] \\
\rightarrow & {\left[\left(0, \ldots, 0, f_{k}\left(s, v_{k}+v_{0}\right), f_{k+1}\left(s, v_{k+1}+v_{0}\right), \ldots\right)\right], }
\end{aligned}
$$

by $v_{0}$ on $\mathcal{A}^{\infty}(X, A)$, is well defined for all $\left[\left(f_{1}, \ldots, f_{k-1}, f_{k}, f_{k+1} \ldots\right)\right] \in \mathcal{A}^{\infty}(X, A)$.

By the uniform equicontinuity condition in the definition of $\mathcal{A}^{\infty}(X, A)$, this translation operator is norm-continuous in $v_{0}$. Hence a $\Gamma$ action on $C(Z) \hat{\otimes} \mathcal{A}^{\infty}(X, A)$ can be defined exactly in the same way as the $\Gamma$ action on $C(Z) \hat{\otimes} \mathcal{A}(X) \hat{\otimes} A$. 
Now we can define the Bott map

$$
\beta^{\infty}: K K_{*}^{\Gamma}\left(\mathcal{E} \Gamma, C(Z) \hat{\otimes} \mathcal{S}^{\infty}(A)\right) \rightarrow K K_{*}^{\Gamma}\left(\mathcal{E} \Gamma, C(Z) \hat{\otimes} \mathcal{A}^{\infty}(X, A)\right),
$$

in a way similar to the definition of the Bott map

$$
\beta: K K_{*}^{\Gamma}(\mathcal{E} \Gamma, C(Z) \hat{\otimes} \mathcal{S} \hat{\otimes} A) \rightarrow K K_{*}^{\Gamma}(\mathcal{E} \Gamma, C(Z) \hat{\otimes} \mathcal{A}(X) \hat{\otimes} A),
$$

where $\mathcal{S}^{\infty}(A)$ is defined in Section 2.

The Bott map $\beta^{\infty}$ is induced by the asymptotic homomorphism

$\left[u \hat{\otimes}\left(f_{1} \hat{\otimes} b_{1}, \ldots, f_{k} \hat{\otimes} b_{k}, \ldots\right)\right] \rightarrow\left[u \hat{\otimes}\left(\left(f_{1}\right)_{t} \hat{\otimes} b_{1}, \ldots,\left(f_{k}\right)_{t} \hat{\otimes} b_{k}, \ldots\right)\right]$,

from $C(Z) \hat{\otimes} \mathcal{S}^{\infty}(A)$ to $C(Z) \hat{\otimes} \mathcal{A}^{\infty}(X, A)$, where $u \in C(Z), f_{k} \in \mathcal{S}, b_{k} \in A$ and $\left(f_{k}\right)_{t}\left(s, v_{k}\right)=f_{k}\left(t^{-1}\left(s, \phi\left(v_{k}\right)\right)\right)$ for all $\left(s, v_{k}\right) \in \mathbb{R} \times V_{k}$.

We claim that the Bott map

$$
\beta^{\infty}: K K_{*}^{\Gamma}\left(\mathcal{E} \Gamma, C(Z) \hat{\otimes} \mathcal{S}^{\infty}(A)\right) \rightarrow K K_{*}^{\Gamma}\left(\mathcal{E} \Gamma, C(Z) \hat{\otimes} \mathcal{A}^{\infty}(X, A)\right)
$$

is an isomorphism.

This claim follows from the standard Mayer-Vietoris and five lemma argument, Corollary 2.8, and the fact that, for any finite subgroup $H$ of $\Gamma, Z$ is $H$-equivariantly homotopy equivalent to a point $\mu_{0} \in Z$ fixed by $H$ (using the linear homotopy). The point $\mu_{0}$ can be obtained by averaging the $H$-orbit of a point in $Z$ using the assumption that $H$ is a finite group and the fact that $Z$ is a convex and compact topological space. Then

$$
g\left[u(\mu) \hat{\otimes}\left(f_{k}\left(\left(s, \phi\left(v_{k}-v_{0}^{k}\right)\right)\right) \hat{\otimes} a_{k}\right)\right]
$$

evaluated at $\mu=\mu_{0}$ is equal to

$$
\left[u(\mu) \hat{\otimes}\left(f_{k}\left(\left(s, \phi\left(v_{k}-v_{0}^{k}\right)\right)\right) \hat{\otimes} g\left(a_{k}\right)\right)\right]
$$

evaluated at $\mu=\mu_{0}$ for all $g \in H, u \in C(Z), f_{k} \in \mathcal{S}, v_{0}^{k} \in V_{k}, s \in \mathbb{R}, v_{k} \in V_{k}, a_{k} \in A$ and $\left[\left(f_{k} \hat{\otimes} a_{k}\right)\right] \in \mathcal{S}^{\infty}(A)$. The last equation follows from the definition of the $\Gamma$ action on $C(Z) \hat{\otimes} \mathcal{A}^{\infty}(X, A)$.

We now consider the commutative diagram

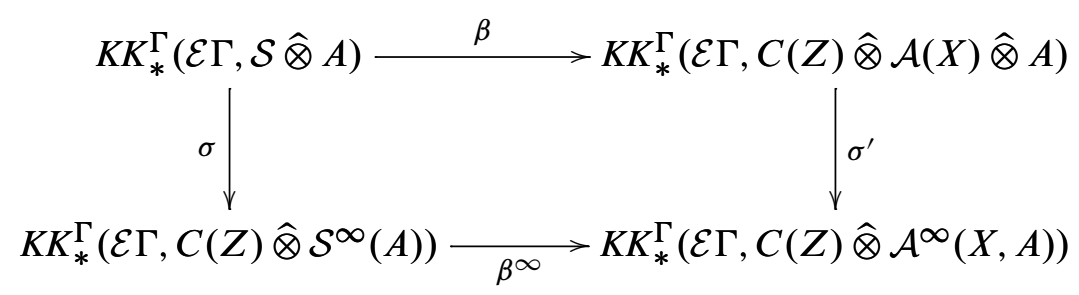


where $\sigma$ is induced by the homomorphism $\mathcal{S} \hat{\otimes} A \rightarrow C(Z) \hat{\otimes} \mathcal{S}^{\infty}(A)$ mapping each element $f$ to $1 \hat{\otimes}\left[\left(f \hat{\otimes} p_{0}\right)\right]$ (here 1 is the constant 1 function on $Z,\left[\left(f \hat{\otimes} p_{0}\right)\right]$ is the element represented by the constant sequence consisting of $f \hat{\otimes} p_{0}$, and $p_{0}$ is a rank one projection of grading degree 0 in $\mathcal{K}$ ) and $\sigma^{\prime}$ is induced by the homomorphism from $C(Z) \hat{\otimes} \mathcal{A}(X) \hat{\otimes} A$ to $C(Z) \hat{\otimes} \mathcal{A}^{\infty}(X, A)$ which maps each element $u \hat{\otimes} f$ to the element represented by the sequence $u \hat{\otimes}\left(f_{k} \hat{\otimes} p_{0}\right)$ (here $u \in C(Z), f \in \mathcal{A}(X) \hat{\otimes} A$, and $f_{k}$ is the restriction of $f$ to $V_{k}$ ).

We observe that $\sigma$ is injective. This can be seen as follows. Let $\tilde{\sigma}$ be the homomorphism

$$
K K_{*}^{\Gamma}(\mathcal{E} \Gamma, \mathcal{S} \hat{\otimes} A) \rightarrow K K_{*}^{\Gamma}\left(\mathcal{E} \Gamma, \mathcal{S}^{\infty}(C(Z) \hat{\otimes} A)\right)
$$

obtained by composing $\sigma$ with the homomorphism from $K K_{*}^{\Gamma}\left(\mathcal{E} \Gamma, C(Z) \hat{\otimes} \mathcal{S}^{\infty}(A)\right)$ to $K K_{*}^{\Gamma}\left(\mathcal{E} \Gamma, \mathcal{S}^{\infty}(C(Z) \hat{\otimes} A)\right)$ that is induced by the inclusion homomorphism from $C(Z) \hat{\otimes} \mathcal{S}^{\infty}(A)$ to $\mathcal{S}^{\infty}(C(Z) \hat{\otimes} A)$. It suffices to prove that $\tilde{\sigma}$ is injective. There exists a natural homomorphism

$$
\prod K K_{*+1}^{\Gamma}(\mathcal{E} \Gamma, C(Z) \hat{\otimes} A) \rightarrow K K_{*}^{\Gamma}\left(\mathcal{E} \Gamma, \mathcal{S}^{\infty}(C(Z) \hat{\otimes} A)\right),
$$

where $\prod K K_{*+1}^{\Gamma}(\mathcal{E} \Gamma, C(Z) \hat{\otimes} A)$ is defined to be the inductive limit of the product $\prod K K_{*+1}^{\Gamma}(\Delta, C(Z) \hat{\otimes} A)$ over all $\Gamma$-cocompact subsets $\Delta$ of $\mathcal{E} \Gamma$. This homomorphism induces a homomorphism (denoted by $\tau$ )

$$
\begin{aligned}
&\left(\prod K K_{*+1}^{\Gamma}(\mathcal{E} \Gamma, C(Z) \hat{\otimes} A)\right) /\left(\bigoplus K K_{*+1}^{\Gamma}(\mathcal{E} \Gamma, C(Z) \hat{\otimes} A)\right) \longrightarrow \\
& K K_{*}^{\Gamma}\left(\mathcal{E} \Gamma, \mathcal{S}^{\infty}(C(Z) \hat{\otimes} A)\right) .
\end{aligned}
$$

Proposition 2.6, together with a standard Mayer-Vietoris and five lemma argument, implies that $\tau$ is an isomorphism. There is also a suspension isomorphism (denoted by $\theta)$ from $K K_{*}^{\Gamma}(\mathcal{E} \Gamma, \mathcal{S} \hat{\otimes} A)$ to $K K_{*+1}^{\Gamma}(\mathcal{E} \Gamma, A)$. Let $\varsigma$ be the homomorphism

$$
K K_{*+1}^{\Gamma}(\mathcal{E} \Gamma, A) \rightarrow\left(\prod K K_{*+1}^{\Gamma}(\mathcal{E} \Gamma, C(Z) \hat{\otimes} A)\right) /\left(\bigoplus K K_{*+1}^{\Gamma}(\mathcal{E} \Gamma, C(Z) \hat{\otimes} A)\right)
$$

which is obtained as the composition of the homomorphism from $K K_{*+1}^{\Gamma}(\mathcal{E} \Gamma, A)$ to $K K_{*+1}^{\Gamma}(\mathcal{E} \Gamma, C(Z) \hat{\otimes} A)$ induced by the inclusion map from $A$ to $C(Z) \hat{\otimes} A$ with the group homomorphism mapping each element $z \in K K_{*+1}^{\Gamma}(\mathcal{E} \Gamma, C(Z) \hat{\otimes} A)$ to the element in $\left(\prod K K_{*+1}^{\Gamma}(\mathcal{E} \Gamma, C(Z) \hat{\otimes} A)\right) /\left(\bigoplus K K_{*+1}^{\Gamma}(\mathcal{E} \Gamma, C(Z) \hat{\otimes} A)\right)$ represented by the constant sequence consisting of $z$. By contractibility of $Z$, we know that $\varsigma$ is injective. We have

$$
\tilde{\sigma}=\tau \circ \varsigma \circ \theta .
$$

Now the injectivity of $\widetilde{\sigma}$ follows from the injectivity of $\varsigma$ and the fact that $\tau$ and $\theta$ are isomorphisms. 
Finally Proposition 4.1 follows from the injectivity of $\sigma$, the claim that $\beta^{\infty}$ is an isomorphism, and the commutative diagram in this proof.

\section{Proof of the main result}

In this section, we give the proof of Theorem 1.2, the main result of this paper. The proof of Theorem 1.3 is essentially similar.

Proof Let $A$ be any $\Gamma-C^{*}$-algebra. We consider the commutative diagram

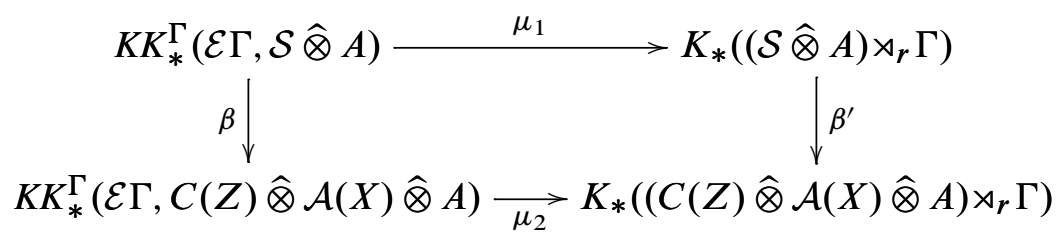

where $\mu_{1}$ and $\mu_{2}$ are the Baum-Connes assembly maps, $\beta$ and $\beta^{\prime}$ are respectively the homomorphisms induced by the inclusion $\mathbb{C} \rightarrow C(Z)$ composed with the Bott maps defined in Section 4. The commutativity of the above diagram follows from the definitions of the Bott maps and the relation between the Baum-Connes map and the evaluation map (compare with the discussion after Corollary 3.7 at the end of Section 3).

Notice that the inclusion map $\mathbb{C} \rightarrow C(Z)$ induces an isomorphism

$$
K K_{*}^{\Gamma}(\mathcal{E} \Gamma, \mathcal{S} \hat{\otimes} A) \rightarrow K K_{*}^{\Gamma}(\mathcal{E} \Gamma, C(Z) \hat{\otimes} \mathcal{S} \hat{\otimes} A) .
$$

This, together with Proposition 4.1, implies that the map $\beta$ is injective. The fact that $C(Z) \hat{\otimes} \mathcal{A}(X) \hat{\otimes} A$ is a $\Gamma$-proper $C^{*}$-algebra implies that $\mu_{2}$ is an isomorphism (it can be seen by the Mayer-Vietoris and five lemma argument; see Guentner-HigsonTrout [6]). The above facts, together with commutativity of the above diagram, imply that $\mu_{1}$ is injective. It follows that the Baum-Connes assembly map $\mu$ in Theorem 1.2 is injective.

\section{References}

[1] MF Atiyah, Bott periodicity and the index of elliptic operators, Quart. J. Math. Oxford Ser. 19 (1968) 113-140 MR0228000

[2] P Baum, A Connes, N Higson, Classifying space for proper actions and $K$-theory of group $C^{*}$-algebras, from: " $C^{*}$-algebras: 1943-1993 (San Antonio, TX, 1993)", Contemp. Math. 167, Amer. Math. Soc., Providence, RI (1994) 240-291 MR1292018 
[3] Y Benyamini, J Lindenstrauss, Geometric nonlinear functional analysis Vol. 1, American Mathematical Society Colloquium Publications 48, American Mathematical Society (2000) MR1727673

[4] N Brown, E Guentner, Uniform embeddings of bounded geometry spaces into reflexive Banach space, Proc. Amer. Math. Soc. 133 (2005) 2045-2050 MR2137870

[5] M Gromov, Asymptotic invariants of infinite groups, from: "Geometric group theory, Vol. 2 (Sussex, 1991)”, London Math. Soc. Lecture Note Ser. 182, Cambridge Univ. Press (1993) 1-295 MR1253544

[6] E Guentner, N Higson, J Trout, Equivariant E-theory for $C^{*}$-algebras, Mem. Amer. Math. Soc. 148 (2000) viii+86 MR1711324

[7] N Higson, Bivariant $K$-theory and the Novikov conjecture, Geom. Funct. Anal. 10 (2000) 563-581 MR1779613

[8] N Higson, G Kasparov, J Trout, A Bott periodicity theorem for infinite-dimensional Euclidean space, Adv. Math. 135 (1998) 1-40 MR1617411

[9] W B Johnson, N L Randrianarivony, $l_{p}(p>2)$ does not coarsely embed into a Hilbert space, Proc. Amer. Math. Soc. 134 (2006) 1045-1050 MR2196037

[10] G Kasparov, G Skandalis, Groups acting properly on "bolic" spaces and the Novikov conjecture, Ann. of Math. 158 (2003) 165-206 MR1998480

[11] M Mendel, A Naor, Metric cotype, Ann. of Math. 168 (2008) 247-298 MR2415403

[12] J Roe, Coarse cohomology and index theory on complete Riemannian manifolds, Mem. Amer. Math. Soc. 104 (1993) x+90 MR1147350

[13] G Skandalis, J L Tu, G Yu, The coarse Baum-Connes conjecture and groupoids, Topology 41 (2002) 807-834 MR1905840

[14] G Yu, Localization algebras and the coarse Baum-Connes conjecture, $K$-Theory 11 (1997) 307-318 MR1451759

[15] G Yu, The coarse Baum-Connes conjecture for spaces which admit a uniform embedding into Hilbert space, Invent. Math. 139 (2000) 201-240 MR1728880

GK: Department of Mathematics, 1326 Stevenson Center, Vanderbilt University Nashville TN 37240, USA

GY: Department of Mathematics, Texas A\&M University

College Station TX 77843, USA

GY: Shanghai Center for Mathematical Sciences, Fudan University

Shanghai, China

gennadi.kasparov@vanderbilt.edu, guoliang.yu@vanderbilt.edu

Proposed: Steve Ferry

Seconded: Ralph Cohen, Yasha Eliashberg
Received: 21 February 2010

Revised: 30 March 2011 